\title{
Influencia del tratamiento de oxidación de la aleación MA956 sobre sus propiedades mecánicas
}

\author{
J. Chao ${ }^{(*)}$ y J. Ibáñez ${ }^{(*)}$
}

\begin{abstract}
Resumen La aleación MA956 es un material ferrítico fabricado por aleado mecánico que presenta la peculiaridad de generar mediante un proceso de oxidación térmica una delgada capa de alúmina $\alpha$ densa y bien adherida al substrato que protege al material contra los ambientes agresivos. En este trabajo se estudia la influencia del tratamiento de oxidación $\left(1.100{ }^{\circ} \mathrm{C} / 100 \mathrm{~h}\right)$ sobre el comportamiento mecánico de esta aleación a temperatura ambiente. En particular, se observa que en tracción y en compresión, la tensión de flujo plástico del material con capa es inferior a la del material sin capa. Dicha disminución de la tensión de flujo se puede atribuir a la formación, durante el proceso de oxidación, de dislocaciones en arista en una estrecha franja de substrato adyacente a la capa.
\end{abstract}

Palabras clave: Propiedades mecánicas. Capa de óxido. Aleación MA956.

\section{Influence of the oxidation treatment on the mechanical properties of the MA956 alloy}

\begin{abstract}
The MA956 alloy is a ferritic oxide dispersion strengthened alloy processed by mechanical alloying which exhibits a good high temperature oxidation resistance due to the capability to develop a compact and tightly adherent $\alpha-\mathrm{Al}_{2} \mathrm{O}_{3}$ scale by thermal oxidation. The present study deals with the effects of the oxidation treatment $\left(1,100{ }^{\circ} \mathrm{C} / 100 \mathrm{~h}\right)$ on its mechanical behavior at room temperature. It has been found that the oxide scale induces a decrease of the flow strength of the $\mathrm{MA} 956 / \mathrm{Al}_{2} \mathrm{O}_{3}$ system with respect to that of scale-free specimens. This feature has been related to the formation during the preoxidation treatment of a high density of edge dislocations in a narrow zone of the substrate beneath the scale.
\end{abstract}

Keywords: Mechanical properties. Oxide scale. MA956 alloy.

\section{INTRODUCCIÓN}

La aleación MA 956 es un material de base hierro reforzado con dispersoides de itria, diseñada para trabajar a alta temperatura, y que tiene como principal característica la formación durante su exposición a alta temperatura $\left(1.100^{\circ} \mathrm{C}\right)$ de una capa de alúmina $\alpha$ densa y bien adherida que la protege contra la oxidación. Estudios anteriores han mostrado que dicha capa ofrece una buena resistencia a la corrosión a temperatura ambiente frente a los fluidos orgánicos, que la hace interesante como un posible biomaterial (1). No obstante, debido al

(*) Centro Nacional de Investigaciones Metalúrgicas, CENIM (CSIC), Avda. de Gregorio del Amo 8, 28040-Madrid (España). campo de aplicación para el cual ha sido diseñado el material, su comportamiento mecánico a temperatura ambiente no ha sido estudiado extensamente. En trabajos anteriores ( 2 y 3 ), se ha mostrado que la curva del ensayo de tracción de la aleación preoxidada está desplazada hacia valores de la tensión más bajos con respecto a la de la misma aleación preoxidada, pero en la que se eliminó previamente la capa. Tomando como referencia la magnitud (4.100 MPa) de las tensiones residuales de compresión generadas en la capa de alúmina durante el tratamiento de oxidación de una aleación muy similar a la MA956 (4), se predijo, mediante un simple análisis mecánico, que las correspondientes tensiones residuales de tracción en el substrato podrían ser las responsables del ablandamiento observado en el material con capa. Según dicho análisis, en un 
ensayo de compresión, la tensión de flujo del material sin capa debería ser inferior a la del material con capa.

El objetivo de este trabajo es verificar si los resultados de los ensayos de compresión son congruentes con las conclusiones alcanzadas con los correspondientes de tracción arriba indicados y analizar el efecto del tratamiento de oxidación sobre el comportamiento mecánico de la aleación MA956.

\section{MÉTODO EXPERIMENTAL}

\subsection{Material}

El material lo suministró el fabricante como laminado en caliente en forma de barra de $9,5 \mathrm{~mm}$ de diámetro. La composición química de la aleación es $20 \mathrm{Cr}-4,5 \mathrm{Al}-0,5 \mathrm{Ti}-0,5 \mathrm{Y}_{2} \mathrm{O}_{3}$ (\% en masa), resto hierro. La microestructura, de difícil resolución, está compuesta por granos muy finos, alargados y entrelazados (5). En dicho estado, la orientación cristalográfica de los granos está definida por las direcciones $<110>$ y $<100>(6)$. El tratamiento para generar la capa de alúmina consiste en un calentamiento isotérmico a $1.100{ }^{\circ} \mathrm{C}$ al aire, durante $100 \mathrm{~h}$. Dicho tratamiento, además de generar una capa de $5 \mu \mathrm{m}$ de espesor, densa y bien adherida, modifica la microestructura del material en dos aspectos relevantes. En primer lugar, el grano crece, particularmente en el sentido longitudinal de la barra, hasta alcanzar $20 \mathrm{~mm}$ de longitud y $150 \mu \mathrm{m}$ de diámetro (2). En segundo lugar, los granos se reorientan, de forma que la dirección $\langle 100\rangle$ coincide con la dirección longitudinal de la barra (6). A nivel submicroscópico, dicho tratamiento produce, salvo en una estrecha franja superficial afectada por la oxidación, una microestructura con una densidad de dislocaciones muy baja (7), ya que dicho tratamiento de oxidación produce una recristalización del substrato. En las proximidades de la superficie del substrato se produce, como consecuencia del proceso de oxidación, una microestructura con una densidad de dislocaciones elevada (8 y 9).

\subsection{Ensayos mecánicos}

Las probetas de compresión eran cilíndricas con $3,5 \mathrm{~mm}$ de diámetro y $6,5 \mathrm{~mm}$ de altura. Los extremos de dichas probetas fueron rectificados de forma que las caras fueran plano-paralelas y perpendiculares al eje de la probeta. Los ensayos se realizaron a una velocidad de deformación inicial de $10^{-4} \mathrm{~s}^{-1}$. Las diferencias microestructurales entre las probetas de compresión designadas como con capa y sin capa es que en estas últimas, además de no poseer capa, tampoco tienen la maraña de dislo- caciones arriba mencionada, siendo, por lo demás, idénticas, ya que han recibido el mismo tratamiento. Para eliminar la capa deformada que se ha producido como consecuencia del mecanizado en las probetas designadas como sin capa, dichas probetas, fueron atacadas químicamente durante $15 \mathrm{~s}$ con el reactivo que se utiliza para revelar la microestructura.

Los ensayos de dureza Vickers se realizaron con una carga de $200 \mathrm{~g}$. Para proteger la capa durante la preparación metalográfica y poder situar las huellas lo más próximo posible a la intercara $\mathrm{Al}_{2} \mathrm{O}_{3} /$ MA956 se recubrió electrolíticamente la muestra (previa deposición de una película de oro que hiciera la muestra eléctricamente conductora) con una capa de cobre de $200 \mu \mathrm{m}$ de espesor.

\section{RESULTADOS EXPERIMENTALES}

Los resultados de los ensayos de tracción y de compresión a temperatura ambiente se representan conjuntamente, en función del tipo de esfuerzo (tracción ó compresión), en la figura 1(a) y en función de la presencia de la capa (con capa ó sin capa) en la figura 1(b). Con objeto de hacer comparables estos resultados, se presentan en la forma de tensión real frente a deformación real. En la figura 1(a), se observa que, independientemente de que el esfuerzo fuera de tracción ó de compresión, el efecto de la capa es reducir la tensión de flujo plástico. La magnitud de esta reducción es aproximadamente de unos $25 \mathrm{MPa}$. En la figura 1(b), se observa, tanto para el material con capa como sin capa, que el endurecimiento y la tensión de flujo plástico en compresión es mayor que en tracción.

En la figura 2 se muestra cómo varía la dureza con la distancia a la intercara capa-substrato en la región con capa y en una región en la que se produjo un desconchamiento local de la misma. Comparando la variación de dureza en ambas regiones se observa que el endurecimiento del substrato en la región con capa se debe al constreñimiento de la deformación plástica del substrato inducido por la capa y no a un cambio microestructural.

\section{DISCUSIÓN}

El tratamiento de oxidación genera tensiones internas en el sistema capa-substrato. Durante la etapa de formación de la capa, se generan tensiones residuales de compresión en la capa y de tracción en el substrato, debido a que el volumen del óxido formado es mayor que el del metal consumido. Posteriormente, durante la etapa de enfriamiento desde la temperatura de oxidación a la temperatura ambiente, se genera un sistema similar de tensiones 


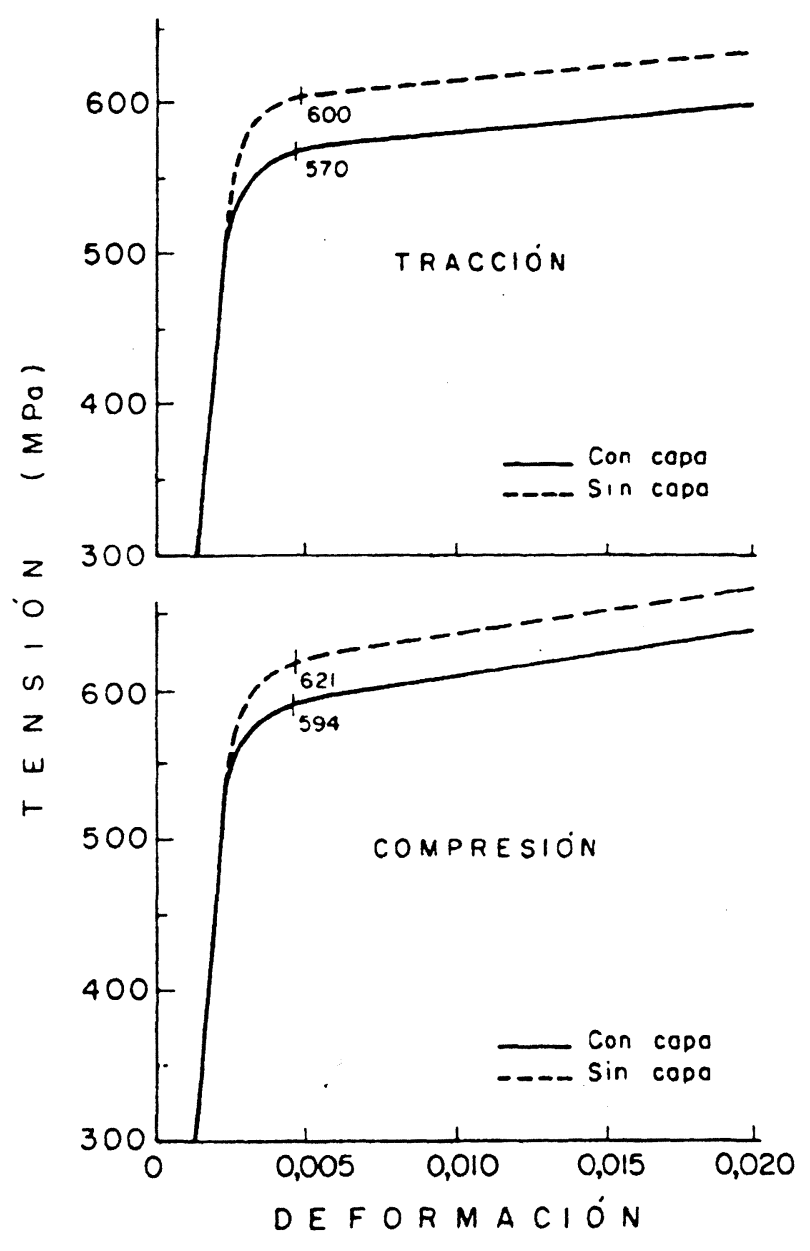

(a)

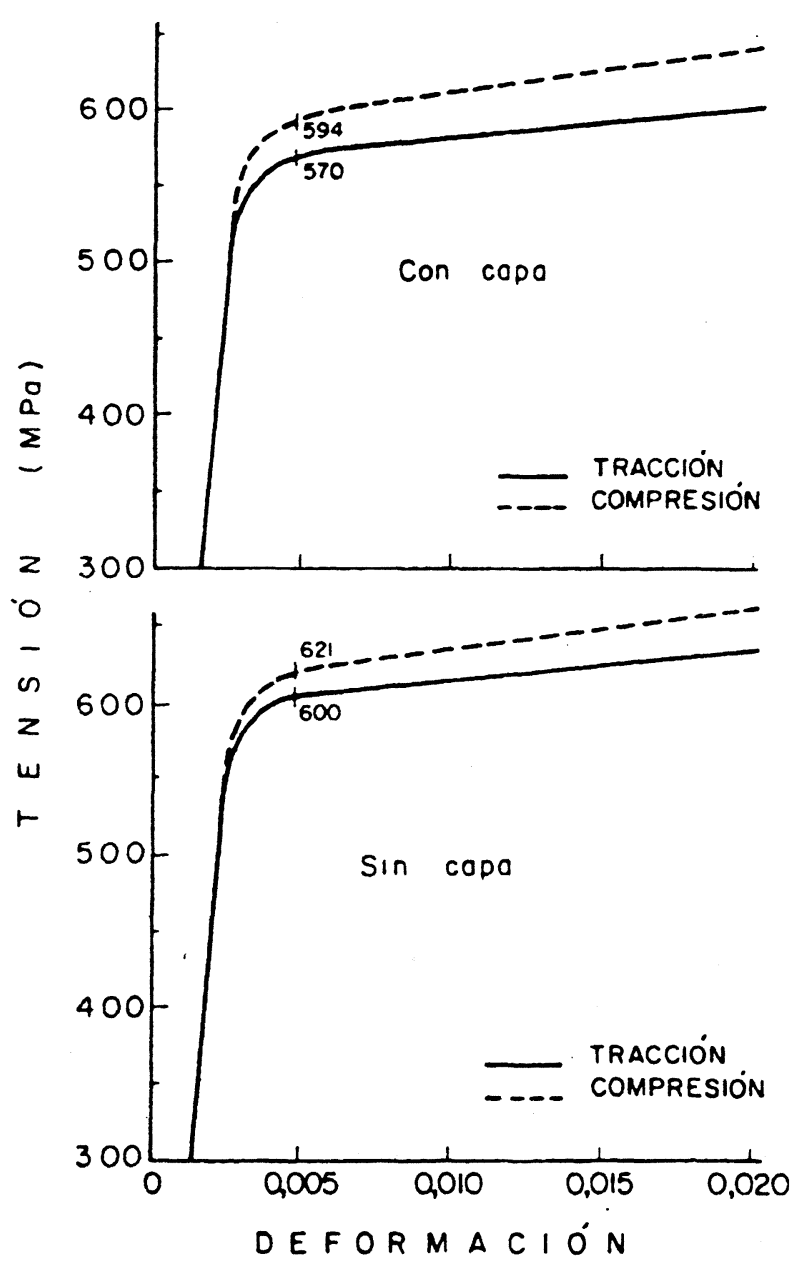

(b)

FIG. 1.- Comparación de las curvas tensión real vs deformación real de los ensayos de tracción y de compresión a temperatura ambiente de la aleación preoxidada. a) Efecto de la capa. $b$ ) Efecto del sentido del esfuerzo.

FIG. 1.- Room temperature tensile and compressive flow curves. a) Scale effect. b) Stress direction effect.

como consecuencia de la diferencia en el coeficiente de expansión térmica de la alúmina $\left(8 \times 10^{-6}{ }^{\circ} \mathrm{C}^{-1}\right)$ y el del substrato $\left(14 \times 10^{-6}{ }^{\circ} \mathrm{C}^{-1}\right)$. La resultante de estos sistemas de tensiones residuales daría lugar a un ablandamiento o a un endurecimiento aparente del sistema capa-substrato, según fuera el ensayo de tracción o de compresión, respectivamente. Las curvas tensión-deformación de los ensayos de tracción y de compresión de la figura 1(a) muestran, sin embargo, que, en ambos casos, el efecto de la capa es reducir la tensión de flujo. Parece, entonces, que el efecto que pudieran producir las tensiones residuales sobre la tensión de flujo está relegado a un papel secundario. Por otra parte, la figura 1(b) muestra, tanto para el material con capa como sin capa, que la tensión de flujo plástico en compresión es mayor que en tracción. Este efecto, conocido en la literatura como "efecto de resistencia diferencial" (10), podría atribuirse en esta aleación a las tensiones internas inducidas por los dispersoides de itria
(3) y al propio proceso de fabricación de la aleación.

El efecto más común que produce la presencia de una delgada capa de un material más rígido es endurecer el sistema capa-substrato en relación al substrato. En general, la magnitud de tal endurecimiento no se corresponde con la que cabría esperar considerando el sistema capa-substrato como un material compuesto, sino que es considerablemente mayor. Para explicar este comportamiento se han propuesto varios mecanismos (11-17), que, si bien presentan diferencias significativas entre ellos, están basados en que la deformación de un sólido homogéneo se inicia en su superficie. El efecto endurecedor de la capa es, entonces, bloquear la deformación plástica del substrato. Ello implicaría, por otra parte, que la capa debe permanecer bien adherida al substrato.

En nuestro caso, resulta evidente, de la comparación de las distribuciones de dureza en la regiones 


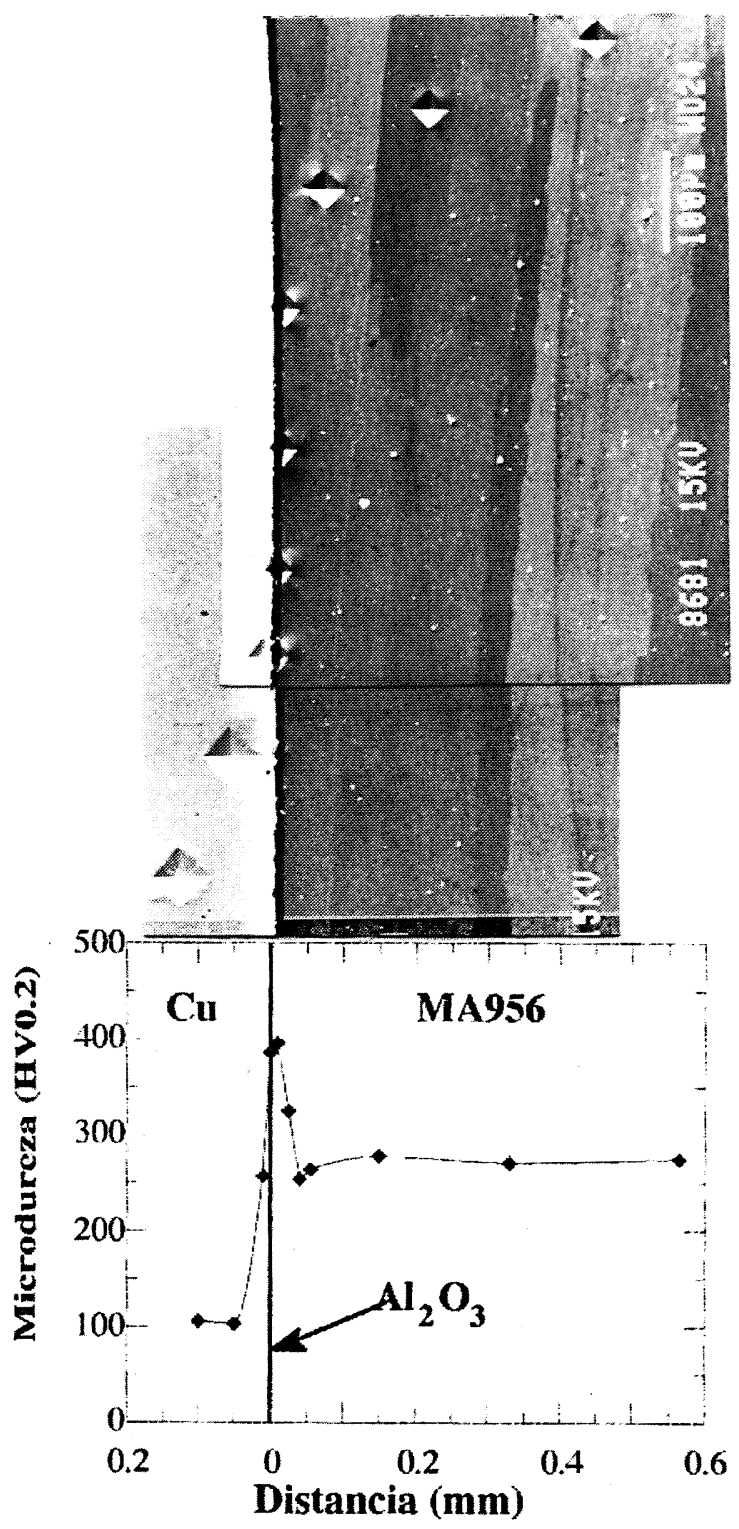

(a)

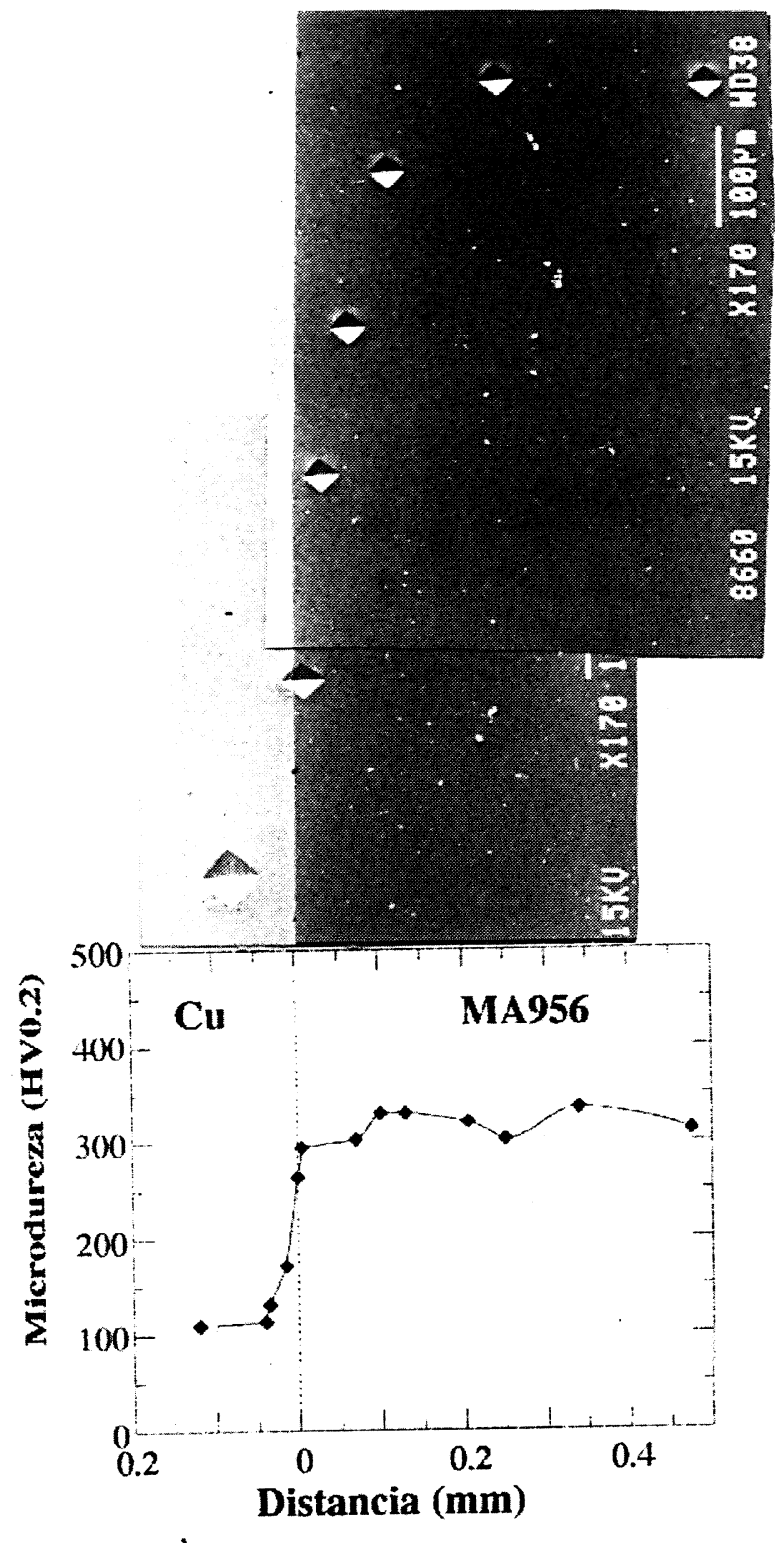

(b)

FIG. 2.- Variación de la dureza con la distancia a la intercara capa-substrato. a) Región con capa. b) Región sin capa.

FIG. 2.- Variation of HV0.2 microhardness with normalized distance from the scale-metal interface. a) Zone with scale. b) Zone without scale.

con capa y sin capa de la figura 2, que ésta ejerce un efecto bloqueante sobre la deformación plástica del substrato y, por tanto, produce un efecto endurecedor.

Con menos frecuencia, y normalmente a temperaturas criogénicas, se ha observado un ablandamiento inducido por la capa. La causa de este ablandamiento se ha relacionado con el efecto que produce el proceso de formación de la capa sobre la estructura de dislocaciones en las inmediaciones de la intercara. En el caso de materiales con estructura cristalina cúbica centrada (18 y 19), se ha atribuido dicho ablandamiento a la generación de dislocacio- nes en arista, las cuales, a temperaturas criogénicas presentan una mayor movilidad que las dislocaciones helicoidales. Sin embargo, conforme se incrementa la temperatura de ensayo, la magnitud del ablandamiento va siendo menor, llegando incluso a manifestarse cierto endurecimiento (18) a temperaturas próximas a la ambiente, en la cual la deformación plástica pasaría a estar controlada por el movimiento de dislocaciones helicoidales (20). En otros casos (21 y 22), se ha sugerido que el ablandamiento inducido por la capa está asociado a la generación de dislocaciones libres. 
De acuerdo con estos modelos, el ablandamiento producido por la capa es microestructural e intrínseco al material y, por lo tanto, no debería depender del tipo de ensayo ni del tamaño de probeta, lo cual concordaría con los resultados experimentales que se muestran en la figura 1(a). Por otra parte, en el caso de la alearión MA956 preoxidada, si bien se conoce que existe una elevada densidad de dislocaciones cerca de la intercara (9) nada se sabe sobre su naturaleza. Con el fin de comprobar indirectamente si son las dislocaciones en arista o las dislocaciones libres las responsables del ablandamiento observado, se ha realizado una serie de ensayos previos sobre probetas de compresión de las mismas dimensiones, tanto del material con capa como sin capa, a $77 \mathrm{~K}$. En el caso de que fueran dislocaciones libres las responsables del ablandamiento, dicho ablandamiento sería similar a temperatura ambiente y a 77 $\mathrm{K}$, mientras que si lo fueran las dislocaciones en arista, y debido a la mayor movilidad que presentan estas dislocaciones a bajas temperaturas, cabría esperar un mayor ablandamiento a $77 \mathrm{~K}$. Los resultados que se han obtenido indican que, a $77 \mathrm{~K}$, el ablandamiento es cuatro veces superior al que se produce a temperatura ambiente. Parece, entonces, que las dislocaciones cerca de la intercara son predominantemente del tipo de arista y, por tanto, el análisis de Sethi y Gibala (18 y 19) resulta más adecuado para describir el efecto de la capa de alúmina en el comportamiento mecánico de la aleación MA 956. Es importante también resaltar que a temperatura ambiente el efecto microestructural inducido por la capa de alúmina predomina sobre el efecto endurecedor asociado al constreñimiento plástico que ejerce la capa y al efecto que las tensiones residuales generadas durante el proceso de oxidación pudieran ejercer sobre la curva de ensayo. En la actualidad, se está realizando un programa de ensayos a diferentes temperaturas con el objeto de determinar el intervalo de temperaturas sobre el que predomina uno u otro efecto y tratar de cuantificarlo.

\section{CONCLUSIONES}

Se ha estudiado el efecto que produce una capa de alúmina sobre el comportamiento mecánico a tracción y compresión de la aleación MA 956, habiéndose obtenido las siguientes conclusiones:

1. La presencia de una capa de alúmina reduce la tensión de flujo plástico de la aleación MA 956 en una magnitud que es independiente de que el ensayo sea de tracción o de compresión. El ablandamiento producido por la capa aumenta conforme disminuye la temperatura de ensayo.

2. El efecto de ablandamiento inducido por la capa puede explicarse cualitativamente por la presen- cia de dislocaciones, predominantemente de tipo arista, situadas en las inmediaciones de la intercara, las cuales habrían sido generadas durante el proceso de oxidación.

3. Este mecanismo predomina sobre el efecto endurecedor asociado a la acción bloqueante de la deformación plástica que ejerce la capa y a los efectos que ejercen las tensiones residuales que se generan durante el tratamiento de oxidación sobre la curva de ensayo.

\section{Agradecimiento}

Este trabajo ha sido financiado por la CICYT. Proyecto MAT95-0796.

\section{REFERENCIAS}

(1) Escudero, M.L. y Gonzalez-Carrasco, J.L. Biomaterials, 15, 1994, 1175.

(2) Chao, J،, Gonzalez-Carrasco, J.L., Ibañez, J., Escudero, M.L. y GonZalez-Doncel, G. Metall. Mater. Trans., 1996, 27A, 3809.

(3) Chao, J. y Gonzalez-Carrasco, J.L. Mater. Sci. Technol., en prensa.

(4) Banks, J.P., Gohil, D.D., Evans, H.E., Hall, D.J. y SAUnders, S.R.J. Materials for Advanced Power Engineering, Part II, Editado por D. Coutsouradis, J.H. Davidson, J. Ewald, P. Greenfield, T. Khan, M. Malik, D.B. Meadowcroft, V. Regis, R.B. Scarlin, F. Schubert y D.V. Thornton. Klüwer Academic Publishers. 1994. 1543.

(5) Alamo, A., Regle, H., Pons, G. y Bechade, J.L. Mater. Sci. Forum, 1992, 88/90, 183.

(6) Chao, J., Cristina, M.C., Gonzalez-Carrasco, J.L. y Gonzalez-Doncel, G. "VII Cong. Nacional de Ciencia y Tec. Metal.”. CENIM. 1998.

(7) Dubiel, B., Wrobel, M., Ennis, P.J. y CzyrskaFilemonowicz, A. Scripta Mat., 1997, 37, 8, 1215.

(8) Pieragi, B., RapP, R.A. y Hirth, J.P. Oxid. Met., 1995, $44,1 / 2,63$

(9) Ramanarayanan, T.A., Raghavan, M. y PetkovicLuton, R. J. Electrochem. Soc., 1984, 131, 923.

(10) HirTh, J.P. y Cohen, M. Metall. Trans., 1970, 1,3.

(11) Menter, J.W. y Hall, E.O. Nature, 1950, 15, 611

(12) Phillips, D.J. y Thompson, N. Proc. Phys. Soc. Lond. B, 1950, 63, 839 .

(13) FiSHER, J.C. Trans. AIME, 1952, 194, 531.

(14) Westwood, A.R.C. Phil. Mag., 1960, 5, 981.

(15) Nakada, Y. y Chalmers, B. Trans. AIME, 1964, 230, 1339.

(16) Pridans, J. y Bilello, J.C. Acta Metall., 1972, 20, 1339.

(17) Johnson, R.M. y BLOCK, R.J. Acta Metall., 1968, 16, 831.

(18) Sethi, V.K. y Gibala, R. Acta Metall., 1977, 25, 321.

(19) Sethi, V.K. y Gibala, R. Thin Solid Films, 1976, 39, 79.

(20) Bowen, D.K., Christian, J.W. y Taylor, G. Can. J. Phys., 1967, 45, 903.

(21) Ruddle, G.E. y Wilsdorf, H.G.F. Appl. Phys. Letters, 1968, 12, 271

(22) Noebe, R.D. y Gibala, R. Scripta Metall., 1986, 20, 1365. 